\title{
Mott transition in the asymmetric Hubbard model at half-filling within dynamical mean-field theory
}

\author{
Ihor V. Stasyuk and Orest B. Here* \\ Institute for Condensed Matter Physics of the National Academy of Sciences of Ukraine, \\ 1 Svientsitskii Str., 79011 Lviv, Ukraine
}

(Dated: April 12, 2005)

\begin{abstract}
We apply the approximate analytic methods to the investigation of the band structure of the asymmetric Hubbard model where the chemical potentials and electron transfer parameters depend on the electron spin (type of quasiparticles). The Hubbard-I and alloy-analogy approximations are the simplest approximations which are used. Within the alloy-analogy approximation, the energy band of particles does not depend on the transfer parameter of particles of another sort. It means that the gap in the spectrum opens at the critical value $U_{\mathrm{c}}$ that is the same in two different limiting cases: the Falicov-Kimball model and the standard Hubbard model. The approximate analytic scheme of the dynamical mean-field theory is developed to include into the theory the scattering of particles responsible for the additional mechanism (due to the transfer of particles of another sort) of the band formation. We use the so-called GH3 approach that is a generalization of the Hubbard-III approximation. The approach describes the continuous Mott transition with the $U_{\mathrm{c}}$ value dependent on a ratio of transfer parameters of different particles.

PACS numbers: 71.10.Fd, 71.27.+a, 71.30.+h
\end{abstract}

\section{INTRODUCTION}

The asymmetric Hubbard model is considered as a generalization of the Falicov-Kimball model [1] and the Hubbard model [2]. This model describes a system with two sorts of mobile particles (ions, electrons or quasiparticles) with different hopping integrals and different values of chemical potentials.

The hopping of particles is described by creation and annihilation operators and transfer parameters $t_{i j}^{\sigma}$. The Hamiltonian of the model is

$$
H=\sum_{i} H_{i}+\sum_{i j \sigma} t_{i j}^{\sigma} a_{i \sigma}^{\dagger} a_{j \sigma},
$$

where the singe-site part

$$
H_{i}=-\sum_{\sigma} \mu_{\sigma} n_{i \sigma}+U n_{i \uparrow} n_{i \downarrow}
$$

includes chemical potentials $\mu_{\sigma}$ and a local on-site repulsion $U\left(n_{i \sigma}=a_{i \sigma}^{\dagger} a_{i \sigma}\right)$.

Due to a complexity of the problem the asymmetric Hubbard model is much less investigated than its limiting cases: the Falicov-Kimball and Hubbard models. The model was investigated in the large $U$ limit at halffilling. The effective Hamiltonian corresponding to the anisotropic Heisenberg model was derived [3, 4] and the effective antiferromagnetic interaction was discussed 5]. A phase separation phenomenon was considered in the ground state of the asymmetric Hubbard model [6]. Some progress has been achieved in the case of one-dimensional chains $7,8,9$.

*Electronic address: hera@icmp.lviv.ua
The essential achievement in the theory of the strongly correlated electron systems is connected with the development of the dynamical mean-field theory (DMFT) proposed by Metzner and Vollhardt 10] for the Hubbard model (see also Ref. 11 for a review). This method is based on the assumption that the self-energy is a pure local (a single-site) quantity $\Sigma_{i j}^{\sigma}(\omega)=\Sigma^{\sigma}(\omega) \delta_{i j}$ and it becomes exact in the limit of infinite dimensions [12]. The current growing interest to DMFT is related to the new developing technique combining DMFT and the density functional theory within the local density approximation (LDA). The new approach LDA+DMFT [13] allows one to calculate the electronic structure of real materials correctly taking into account the strong local correlations (see Refs. 14, 15 for reviews).

The Falicov-Kimball and Hubbard models were intensively investigated within DMFT (see reviews Refs. 11, 16). In the Falicov-Kimball model, particle densities of states can be calculated exactly [17, 18, 19, 20, 21, 22]. However, the spectrum of localized particles was investigated mostly at half-filling. In the case of the half-filled Hubbard model, a number of numerical and analytic approximate methods was developed and applied to the investigation of metal-insulator transitions 23, 24, 25, 26, 27, 28, 29, 30, 31, 32, 33].

We use the approximate analytic approach to the investigation of the band structure of the asymmetric Hubbard model in DMFT 34|. This method was developed for the Hubbard model [35, 36] and it is based on a mapping of the problem onto the effective single-site Hamiltonian with the auxiliary Fermi-operators describing the environment of a given site. The approach is based on the equations of motion for Hubbard operators followed by the different time decoupling of the higher order Green's functions. The irreducible parts are separated off using projecting on the basis of fermionic Hubbard oper- 
ators. This approach gives DMFT equations in the approximation that is a generalization of the Hubbard-III approximation and includes as simple specific cases the modified alloy-analogy approximation 37] and the Hubbard-III approximation [38]. We call it the generalized Hubbard-III (GH3) approximation. Recently, an alternative decoupling scheme in an equation of motion approach to the Hubbard model in infinite dimensions has been proposed in Ref. 39 .

This paper is organized as follows. In Section III we review the formalism of DMFT, where particle hopping can be introduced in two different ways: using the coherent potential or the effective single-site Hamiltonian with the auxiliary Fermi-filed [35]. The equation of motion approach with the projecting technique and the differenttime decoupling scheme is described in Section III This approach gives the DMFT equations within the generalized Hubbard-III approximation. In Section IV] simpler approximations to the asymmetric Hubbard model are introduced. In Section $\nabla$ some analytic properties concerning continuous metal-insulator transitions are derived. Our results are discussed in Section VI and concluding remarks are given in Section VII

\section{DYNAMICAL MEAN FIELD THEORY}

The particle Green's function in the Matsubara representation is defined as follows

$$
\begin{gathered}
G_{i j}^{\sigma}\left(\tau-\tau^{\prime}\right)=\left\langle\mathcal{T}_{\tau} a_{i \sigma}^{\dagger}(\tau) a_{j \sigma}\left(\tau^{\prime}\right)\right\rangle \\
G_{i j}^{\sigma}\left(\omega_{n}\right)=\int_{0}^{\beta} G_{i j}^{\sigma}(\tau) \mathrm{e}^{-\mathrm{i} \omega_{n} \tau} \mathrm{d} \tau, \quad \omega_{n}=\frac{\pi(2 n+1)}{\beta}
\end{gathered}
$$

where $\mathcal{T}_{\tau}$ denotes imaginary time ordering, and $\beta=1 / T$ is the inverse temperature.

For the Hamiltonian (1), the particle Green's function can be written as a solution of the Larkin equation [40]:

$$
G_{i j}^{\sigma}\left(\omega_{n}\right)=\Xi_{i j \sigma}\left(\omega_{n}\right)+\sum_{l m} \Xi_{i l \sigma}\left(\omega_{n}\right) t_{l m}^{\sigma} G_{m j}^{\sigma}\left(\omega_{n}\right),
$$

or in momentum space

$$
G_{\sigma}\left(\omega_{n}, \mathbf{k}\right)=\Xi_{\sigma}\left(\omega_{n}, \mathbf{k}\right)+\Xi_{\sigma}\left(\omega_{n}, \mathbf{k}\right) t_{\mathbf{k}}^{\sigma} G_{\sigma}\left(\omega_{n}, \mathbf{k}\right),
$$

where $\Xi_{\sigma}\left(\omega_{n}, \mathbf{k}\right)$ is the total irreducible part; it is connected to the Dyson self-energy by the relation:

$$
\Xi_{\sigma}^{-1}\left(\omega_{n}, \mathbf{k}\right)=\mathrm{i} \omega_{n}+\mu_{\sigma}-\Sigma_{\sigma}\left(\omega_{n}, \mathbf{k}\right)
$$

In the limit of high lattice dimensions $d \rightarrow \infty$, the irreducible part becomes a single-site quantity [12, 41]:

$$
\Xi_{i j \sigma}\left(\omega_{n}\right)=\Xi_{\sigma} \delta_{i j}, \quad \Xi_{\sigma}\left(\omega_{n}, \mathbf{k}\right)=\Xi_{\sigma}\left(\omega_{n}\right)
$$

The function $\Xi_{\sigma}\left(\omega_{n}\right)$ or $\Sigma_{\sigma}\left(\omega_{n}\right)$ is calculated using the auxiliary single-site problem. This problem corresponds to the following replacement

$$
\mathrm{e}^{-\beta H} \rightarrow \mathrm{e}^{-\beta H_{\text {eff }}}=\mathrm{e}^{-\beta H_{0}} \mathcal{T}_{\tau} \exp \left[-\int_{0}^{\beta} \mathrm{d} \tau H_{\text {int }}(\tau)\right]
$$

$$
H_{\text {int }}(\tau)=\int_{0}^{\beta} \mathrm{d} \tau^{\prime} \sum_{\sigma} J_{\sigma}\left(\tau-\tau^{\prime}\right) a_{\sigma}^{\dagger}(\tau) a_{\sigma}\left(\tau^{\prime}\right)
$$

where

$$
H_{0}=H_{i}
$$

and $J_{\sigma}\left(\tau-\tau^{\prime}\right)$ is the coherent potential. This function describes the propagation of a particle in the environment without going through the given site between moments $\tau$ and $\tau^{\prime}$. It is determined self-consistently from the condition that the same irreducible part determines the lattice single-site Green's function

$$
\begin{gathered}
G_{\sigma}\left(\omega_{n}\right)=G_{i i}^{\sigma}\left(\omega_{n}\right)=\frac{1}{N} \sum_{\mathbf{k}} G^{\sigma}\left(\omega_{n}, \mathbf{k}\right), \\
G_{\sigma}\left(\omega_{n}, \mathbf{k}\right)=\frac{1}{\Xi_{\sigma}^{-1}\left(\omega_{n}\right)-t_{\mathbf{k}}^{\sigma}}
\end{gathered}
$$

and the Green's function of the effective single-site problem

$$
G_{\sigma}\left(\omega_{n}\right)=\frac{1}{\Xi_{\sigma}^{-1}\left(\omega_{n}\right)-J_{\sigma}\left(\omega_{n}\right)} .
$$

To investigate the Green's functions in the time representation the analytic continuation from the imaginary to real axis $\left(\mathrm{i} \omega_{n} \rightarrow \omega+\mathrm{i} \varepsilon\right)$ is performed:

$$
G_{\sigma}\left(\omega_{n}\right) \rightarrow G_{\sigma}(\omega)=2 \pi\left\langle\left\langle a_{\sigma} \mid a_{\sigma}^{\dagger}\right\rangle\right\rangle_{\omega}
$$

The self-consistency condition is rewritten in the following form

$$
\begin{aligned}
& G_{\sigma}^{-1}(\omega)=\Xi_{\sigma}^{-1}(\omega)-J_{\sigma}(\omega), \\
& G_{\sigma}(\omega)=\int_{-\infty}^{+\infty} \frac{\rho_{\sigma}^{0}(t) \mathrm{d} t}{\Xi_{\sigma}^{-1}(\omega)-t},
\end{aligned}
$$

where (13) is inserted into (12) and the sum over $\mathbf{k}$ is replaced by the integration with the noninteracting density of states. The infinite dimensional hypercubic lattice and the Bethe lattice are the most popular lattices investigated in the dynamical mean-field theory. A Gaussian noninteracting density of states corresponds to the hypercubic lattice with nearest-neighbour hopping:

$$
\rho_{\sigma}^{\text {hyp }}(\varepsilon)=\frac{1}{t_{\sigma} \sqrt{\pi}} \exp \left(-\frac{\varepsilon^{2}}{t_{\sigma}^{2}}\right)
$$

where the transfer parameters must be scaled to keep the kinetic and interaction energies of the same order of magnitude in the infinite dimensional limit [10]:

$$
t_{i j}^{\sigma}=\frac{t_{\sigma}}{2 \sqrt{d}}
$$

when $i$ and $j$ are nearest-neighbours and zero otherwise. In the case of the Bethe lattice with the coordination 
number $z \rightarrow \infty$, the noninteracting density of states is semielliptic

$$
\rho_{\sigma}^{\text {Bethe }}(\varepsilon)=\frac{1}{2 \pi t_{\sigma}^{2}} \sqrt{4 t_{\sigma}^{2}-\varepsilon^{2}}, \quad|\varepsilon|<2 t_{\sigma},
$$

and the hopping integral for nearest-neighbours is scaled as follows:

$$
t_{i j}^{\sigma}=\frac{t_{\sigma}}{\sqrt{z}} .
$$

Besides the hypercubic and Bethe lattices, the nonsymmetric densities of states can be considered. The infinite dimensional generalization of a face-centred-cubic lattice that was used to describe ferromagnetic order in the Hubbard model [42] is an example of such a lattice.

A solution of the single-site problem (9) gives the particle Green's function $G_{\sigma}(\omega)$ as a functional of the coherent potential $J_{\sigma}(\omega)$. This dependence supplemented by the self-consistency condition forms a closed set of equations for the single-site Green's function and the coherent potential.

We reformulate the single-site problem introducing the effective Hamiltonian as it was done in Ref. 35:

$$
H_{\mathrm{eff}}=H_{0}+\sum_{\sigma} V_{\sigma}\left(a_{\sigma}^{\dagger} \xi_{\sigma}+\xi_{\sigma}^{\dagger} a_{\sigma}\right)+H_{\xi}
$$

The auxiliary fermionic operators $\xi_{\sigma}^{\dagger}, \xi_{\sigma}$ are introduced to describe particle hopping between the site $\left(H_{0}\right)$ and effective environment defined by the Hamiltonian $H_{\xi}$. The coherent potential is expressed as the Green's function in unperturbed Hamiltonian $H_{\xi}$ :

$$
J_{\sigma}(\omega)=2 \pi V_{\sigma}^{2}\left\langle\left\langle\xi_{\sigma} \mid \xi_{\sigma}^{\dagger}\right\rangle\right\rangle_{\omega}^{\xi}
$$

Thus, an explicit form of $H_{\xi}$ is not required to solve the problem.

\section{GENERALIZED HUBBARD-III APPROXIMATION}

We use the single-site Hamiltonian of the asymmetric Hubbard model written in terms of Hubbard operators

$$
H_{0}=-\sum_{\sigma}\left[\mu_{\sigma}\left(X^{\sigma \sigma}+X^{22}\right)\right]+U X^{22}
$$

acting on the basis of single-site states $\left|n_{A}, n_{B}\right\rangle$

$$
\begin{aligned}
|0\rangle & =|0,0\rangle, & |A\rangle & =|1,0\rangle, \\
|2\rangle & =|1,1\rangle, & |B\rangle & =|0,1\rangle .
\end{aligned}
$$

In this case, the particle creation and annihilation operators are expressed as

$$
a_{\sigma}=X^{0 \sigma}+\zeta X^{\bar{\sigma} 2}
$$

and the two-time Green's function $G_{\sigma}(\omega) \equiv 2 \pi\left\langle\left\langle a_{\sigma} \mid a_{\sigma}^{\dagger}\right\rangle\right\rangle_{\omega}$ is expressed as:

$$
\begin{aligned}
G_{\sigma}= & 2 \pi\left[\left\langle\left\langle X^{0 \sigma} \mid X^{\sigma 0}\right\rangle\right\rangle_{\omega}+\zeta\left\langle\left\langle X^{0 \sigma} \mid X^{2 \bar{\sigma}}\right\rangle\right\rangle_{\omega}\right. \\
& \left.+\zeta\left\langle\left\langle X^{\bar{\sigma} 2} \mid X^{\sigma 0}\right\rangle\right\rangle_{\omega}+\left\langle\left\langle X^{\bar{\sigma} 2} \mid X^{2 \bar{\sigma}}\right\rangle\right\rangle_{\omega}\right],
\end{aligned}
$$

where the following notations for sort indices are used: $\bar{\sigma}=B, \zeta=+$ for $\sigma=A$ and $\bar{\sigma}=A, \zeta=-$ for $\sigma=B$.

To calculate the Green's functions in (27), we use the equations of motion for Hubbard operators:

$$
\mathrm{i} \frac{\mathrm{d}}{\mathrm{d} t} X^{0 \sigma(\bar{\sigma} 2)}(t)=\left[X^{0 \sigma(\bar{\sigma} 2)}, H_{\mathrm{eff}}\right] .
$$

The commutators (28) are projected on the subspace formed by fermionic operators $X^{0 \sigma}$ and $X^{\bar{\sigma} 2}$ :

$$
\left[X^{\gamma}, H_{\mathrm{eff}}\right]=\alpha_{1}^{\gamma} X^{0 \sigma}+\alpha_{2}^{\gamma} X^{\bar{\sigma} 2}+Z^{\gamma} .
$$

The operators $Z^{0 \sigma(\bar{\sigma} 2)}$ are defined as orthogonal to the operators from the basic subspace [34, 35, 36]:

$$
\left\langle\left\{Z^{0 \sigma(\bar{\sigma} 2)}, X^{0 \sigma(\bar{\sigma} 2)}\right\}\right\rangle=0 .
$$

Thus, these equations (30) determine the projecting coefficients $\alpha_{i}^{0 \sigma(\bar{\sigma} 2)}$ which are expressed in terms of mean values $\left\langle\xi_{\sigma} X^{p q}\right\rangle$ combined into the following constant

$$
\varphi_{\sigma}=\left\langle\xi_{\bar{\sigma}} X^{\bar{\sigma} 0}\right\rangle+\zeta\left\langle X^{\sigma 2} \xi_{\bar{\sigma}}^{\dagger}\right\rangle
$$

and mean values of the Hubbard operators

$$
A_{p q}=\left\langle X^{p p}+X^{q q}\right\rangle, A_{0 \sigma}=1-n_{\bar{\sigma}}, A_{2 \bar{\sigma}}=n_{\bar{\sigma}} .
$$

This procedure leads to the Green's functions of the $\left\langle\left\langle Z^{0 \sigma(\bar{\sigma} 2)} \mid X^{\sigma 0(2 \bar{\sigma})}\right\rangle\right\rangle$ type. The similar procedure can be applied with respect to the second time argument. As a result, we come to the relations between the components of the Green's function $G_{\sigma}$ and scattering matrix $\hat{P}_{\sigma}$. In a matrix representation, we have

$$
\hat{G}_{\sigma}=\hat{G}_{0}^{\sigma}+\hat{G}_{0}^{\sigma} \hat{P}_{\sigma} \hat{G}_{0}^{\sigma},
$$

where

$$
\hat{G}_{\sigma}=2 \pi\left(\begin{array}{ll}
\left\langle\left\langle X^{0 \sigma} \mid X^{\sigma 0}\right\rangle\right\rangle & \left\langle\left\langle X^{0 \sigma} \mid X^{2 \bar{\sigma}}\right\rangle\right\rangle \\
\left\langle\left\langle X^{\bar{\sigma} 2} \mid X^{\sigma 0}\right\rangle\right\rangle & \left\langle\left\langle X^{\bar{\sigma} 2} \mid X^{2 \bar{\sigma}}\right\rangle\right\rangle
\end{array}\right),
$$

and nonperturbed Green's function $\hat{G}_{0}^{\sigma}$ is

$$
\hat{G}_{0}^{\sigma}=\frac{1}{D_{\sigma}}\left(\begin{array}{cc}
\omega-b_{\sigma} & -\zeta \frac{V_{\sigma}}{A_{2 \sigma}} \varphi_{\sigma} \\
-\zeta \frac{V_{\sigma}}{A_{0 \sigma}} \varphi_{\sigma} & \omega-a_{\sigma}
\end{array}\right)\left(\begin{array}{cc}
A_{0 \sigma} & 0 \\
0 & A_{2 \bar{\sigma}}
\end{array}\right)
$$

where

$$
D_{\sigma}=\left(\omega-a_{\sigma}\right)\left(\omega-b_{\sigma}\right)-\frac{V_{\sigma}^{2}}{A_{0 \sigma} A_{2 \bar{\sigma}}} \varphi_{\sigma}^{2}
$$

$$
a_{\sigma}=-\mu_{\sigma}+\frac{V_{\sigma}}{A_{0 \sigma}} \varphi_{\sigma}, \quad b_{\sigma}=U-\mu_{\sigma}+\frac{V_{\sigma}}{A_{2 \bar{\sigma}}} \varphi_{\sigma} .
$$


The scattering matrix

$$
\begin{aligned}
& \hat{P}_{\sigma}=2 \pi\left(\begin{array}{cc}
A_{0 \sigma}^{-1} & 0 \\
0 & A_{2 \bar{\sigma}}^{-1}
\end{array}\right) \\
& \times\left(\begin{array}{cc}
\left\langle\left\langle Z^{0 \sigma} \mid Z^{\sigma 0}\right\rangle\right\rangle & \left\langle\left\langle Z^{0 \sigma} \mid Z^{2 \bar{\sigma}}\right\rangle\right\rangle \\
\left\langle\left\langle Z^{\bar{\sigma} 2} \mid Z^{\sigma 0}\right\rangle\right\rangle & \left\langle\left\langle Z^{\bar{\sigma} 2} \mid Z^{2 \bar{\sigma}}\right\rangle\right\rangle
\end{array}\right)\left(\begin{array}{cc}
A_{0 \sigma}^{-1} & 0 \\
0 & A_{2 \bar{\sigma}}^{-1}
\end{array}\right)
\end{aligned}
$$

contains the scattering corrections of the second and the higher orders in powers of $V_{\sigma}$. The separation of the irreducible parts in $\hat{P}_{\sigma}$ enables us to obtain the mass operator $\hat{M}_{\sigma}=\left.\hat{P}_{\sigma}\right|_{\text {ir }}$ and the single-site Green's function expressed as a solution of the Dyson equation

$$
\hat{G}_{\sigma}=\left(1-\hat{G}_{0}^{\sigma} \hat{M}_{\sigma}\right)^{-1} \hat{G}_{0}^{\sigma}
$$

The mass operator $\hat{M}_{\sigma}$ is calculated in zero approximation

$$
\hat{M}_{\sigma}=\hat{P}_{\sigma}^{(0)}
$$

where the time correlation functions related by the spectral theorem to the irreducible Green's functions are calculated using procedure of different-time decoupling. In our case it means an independent averaging of the products of $X$ and $\xi$ operators. For example,

$$
\begin{aligned}
& \left\langle\xi_{\sigma}^{\dagger}(t)\left(X^{00}+X^{\sigma \sigma}\right)_{t}\left(X^{00}+X^{\sigma \sigma}\right) \xi_{\sigma}\right\rangle^{\mathrm{ir}} \\
& \quad \approx\left\langle\left(X^{00}+X^{\sigma \sigma}\right)_{t}\left(X^{00}+X^{\sigma \sigma}\right)\right\rangle\left\langle\xi_{\sigma}^{\dagger}(t) \xi_{\sigma}\right\rangle .
\end{aligned}
$$

For simplicity we calculate the correlation functions for Hubbard operators in zero approximation

$$
\begin{aligned}
& \left\langle\left(X^{00}+X^{\sigma \sigma}\right)_{t}\left(X^{00}+X^{\sigma \sigma}\right)\right\rangle \\
& \approx\left\langle\left(X^{00}+X^{\sigma \sigma}\right)^{2}\right\rangle=A_{0 \sigma} .
\end{aligned}
$$

In this example, the following Green's function is reconstructed from the correlation functions using the spectral representation $34,35,36,43]$ :

$$
I(\omega)=A_{0 \sigma}\left\langle\left\langle\xi_{\sigma} \mid \xi_{\sigma}^{\dagger}\right\rangle\right\rangle_{\omega}^{\xi}=\frac{A_{0 \sigma}}{2 \pi V^{2}} J_{\sigma}(\omega) .
$$

Using the above procedure, we can obtain the final expressions for the total irreducible part:

$$
\begin{aligned}
\Xi_{\sigma}^{-1}(\omega)= & {\left[\frac{A_{0 \sigma}}{\omega+\mu_{\sigma}-\Omega_{\sigma}(\omega)}+\frac{A_{2 \bar{\sigma}}}{\omega+\mu_{\sigma}-U-\Omega_{\sigma}(\omega)}\right]^{-1} } \\
& +\Omega_{\sigma}(\omega),
\end{aligned}
$$

where

$$
\Omega_{\sigma}(\omega)=J_{\sigma}(\omega)-\frac{R_{\sigma}(\omega)}{A_{0 \sigma} A_{2 \bar{\sigma}}}+\frac{V_{\sigma} \varphi_{\sigma}}{A_{0 \sigma} A_{2 \bar{\sigma}}}
$$

and

$$
\begin{aligned}
& R_{\sigma}(\omega)=-\frac{\left\langle X^{\sigma \sigma}+X^{\bar{\sigma} \bar{\sigma}}\right\rangle}{2} J_{\bar{\sigma}}\left(\omega+\mu_{\sigma}-\mu_{\bar{\sigma}}\right) \\
& -\frac{\left\langle X^{\sigma \sigma}-X^{\bar{\sigma} \bar{\sigma}}\right\rangle}{2 \pi} \int_{-\infty}^{+\infty} \frac{\mathrm{d} \omega^{\prime} \operatorname{Im} J_{\bar{\sigma}}\left(\omega^{\prime}+\mathrm{i} 0^{+}\right)}{\omega-\omega^{\prime}-\mu_{\bar{\sigma}}+\mu_{\sigma}} \tanh \frac{\beta \omega^{\prime}}{2}
\end{aligned}
$$

$$
\begin{aligned}
& +\frac{\left\langle X^{00}+X^{22}\right\rangle}{2} J_{\bar{\sigma}}\left(U-\mu_{\sigma}-\mu_{\bar{\sigma}}-\omega\right) \\
& -\frac{\left\langle X^{00}-X^{22}\right\rangle}{2 \pi} \int_{-\infty}^{+\infty} \frac{\mathrm{d} \omega^{\prime} \operatorname{Im} J_{\bar{\sigma}}\left(-\omega^{\prime}-\mathrm{i} 0^{+}\right)}{\omega-\omega^{\prime}+\mu_{\bar{\sigma}}+\mu_{\sigma}-U} \tanh \frac{\beta \omega^{\prime}}{2} .
\end{aligned}
$$

The average values of the Hubbard operators are determined using the spectral representation of corresponding Green's functions. The particle density of states is calculated as an imaginary part of the interacting singleparticle Green's function:

$$
\rho_{\sigma}(\omega)=-\frac{1}{\pi} \lim _{\eta \rightarrow 0^{+}} \operatorname{Im} G_{\sigma}(\omega+\mathrm{i} \eta)
$$

giving the concentration

$$
n_{\sigma}=\int_{-\infty}^{+\infty} \frac{\rho_{\sigma}(\omega) \mathrm{d} \omega}{\mathrm{e}^{\beta \omega}+1} .
$$

The Green's functions $\left\langle\left\langle\xi_{\sigma} \mid X^{p q}\right\rangle\right\rangle_{\omega}$ that are required to find $\varphi_{\sigma}$ can be calculated from the exact relation

$$
V_{\sigma}\left\langle\left\langle\xi_{\sigma} \mid X^{p q}\right\rangle\right\rangle_{\omega}=J_{\sigma}(\omega)\left\langle\left\langle a_{\sigma} \mid X^{p q}\right\rangle\right\rangle_{\omega}
$$

derived in Ref. 43. Thus, the approximation given by the equations (44)-(46) we called the GH3 approximation.

We consider below the special case at half-filling $\left(\mu_{A}=\right.$ $\left.\mu_{B}=U / 2, n_{A}=n_{B}=1 / 2\right)$, and due to the particle-hole symmetry we have:

$$
\begin{gathered}
\varphi_{\sigma}=0, \\
\Omega_{\sigma}(\omega)=J_{\sigma}(\omega)+2 J_{\bar{\sigma}}(\omega),
\end{gathered}
$$

and

$$
\Xi_{\sigma}^{-1}(\omega)=\omega-\frac{U^{2}}{4\left[\omega-J_{\sigma}(\omega)-2 J_{\bar{\sigma}}(\omega)\right]} .
$$

For the standard Hubbard model $\left(t_{A}=t_{B}, J_{A}=J_{B}\right)$ the approximate solution of the single-site problem gives the usual Hubbard-III approximation.

In the case of the Falicov-Kimball model $\left(t_{B}=0\right.$, $J_{B}(\omega)=0$ ) at half-filling, GH3 gives the following expression:

$$
G_{B}(\omega)=\frac{\omega-2 J_{A}(\omega)}{\omega^{2}-U^{2} / 4-2 \omega J_{A}(\omega)},
$$

where the coherent potential of itinerant particles $J_{A}$ corresponding to the result of the alloy-analogy approximation is calculated exactly.

\section{OTHER APPROXIMATIONS TO THE ASYMMETRIC HUBBARD MODEL}

\section{A. Hubbard-I approximation}

It was the Hubbard-I approximation [2] which in the simplest way described band forming in the Hubbard 

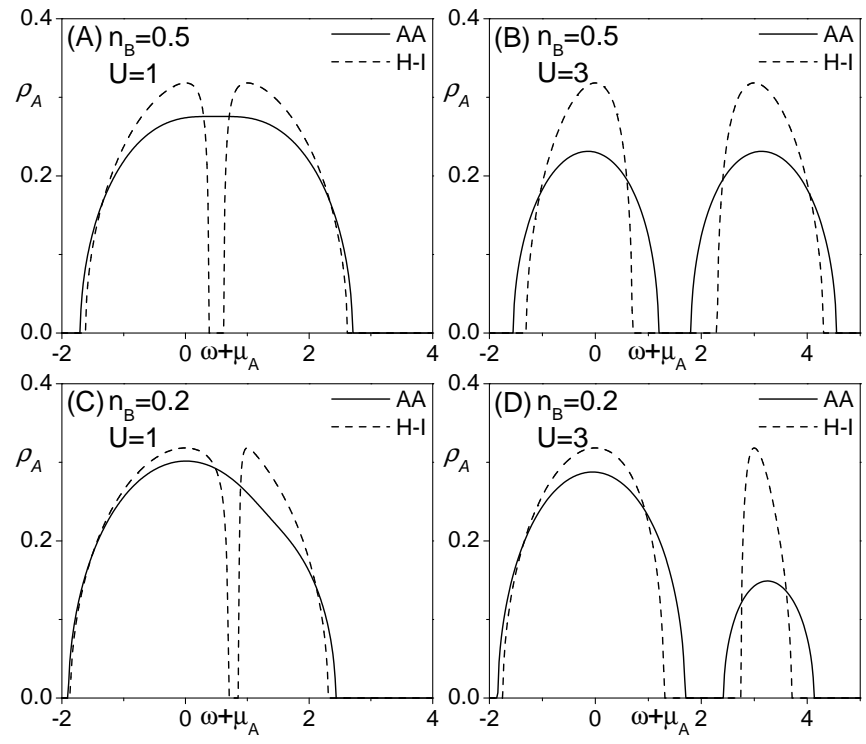

FIG. 1: Density of states $\rho_{A}$ in the alloy-analogy (solid line) and Hubbard-I (dashed line) approximations on the Bethe lattice $\left(t_{A}=1\right)$ for various $n_{B}$ and $U$.

model with the strong on-site repulsion $U$. The total irreducible part in this approximation reads

$$
\Xi_{\sigma}(\omega)=\frac{1-n_{\bar{\sigma}}}{\omega+\mu_{\sigma}}+\frac{n_{\bar{\sigma}}}{\omega+\mu_{\sigma}-U} .
$$

It is well known that the Hubbard-I approximation gives an electron spectrum with the opened gap for any nonzero repulsion $U$ (Fig. 1). Thus, this approximation cannot describe metal-insulator transitions. However, we consider it here to estimate its applicability at large $U$ from a thermodynamic point of view.

The interacting single-site Green's function $G_{\sigma}(\omega)$ is calculated using the integration (17) with a noninteracting density of states $\rho_{\sigma}^{0}(\varepsilon)$. The band of particles of a given type $(\sigma)$ depends here only on $\rho_{\sigma}^{0}(\varepsilon)$ and a concentration of particles of the opposite type, i.e., the transfer parameter $t_{\bar{\sigma}}$ does not have an effect on $G_{\sigma}(\omega)$.

In the large- $U$ limit $\left(U \gg t_{\sigma}\right)$, the density of states has the simple form

$$
\rho_{\sigma}^{\mathrm{H}-\mathrm{I}}(\omega)=\rho_{\sigma}^{0}\left(\frac{\omega+\mu_{\sigma}}{1-n_{\bar{\sigma}}}\right)+\rho_{\sigma}^{0}\left(\frac{\omega+\mu_{\sigma}-U}{n_{\bar{\sigma}}}\right) .
$$

Both spectral subbands are of the same height for any concentration values and are equal to $\rho_{\sigma}^{0}(\varepsilon)$ with the scaled bandwidth.

\section{B. Alloy-analogy approximation}

The system with one type of particles frozen on a lattice can be mapped onto the problem of the electronic structure of simple binary alloys [17, 44]. The alloy-analogy approximation is the single-site solution of the binary alloy problem within the coherent potential approximation [45] that is exact in infinite dimensions. Such a mapping onto the binary alloy is exact for the spinless Falicov-Kimball model with localized ions. Thus, the alloy-analogy approximation exactly describes the band formation by the particle hopping (transfer) and the interaction with the localized particles. However, in the case of the asymmetric Hubbard model this approximation does not include an effect of the transfer of particles of another sort, like the Hubbard-I approximation.

The self-energy part for the Dyson equation in the alloy-analogy approximation reads

$$
\Sigma_{\sigma}(\omega)=\frac{n_{\bar{\sigma}} U}{1-G_{i i}^{\sigma}(\omega)\left(U-\Sigma_{\sigma}(\omega)\right)},
$$

where the single-site Green's function:

$$
\begin{aligned}
G_{i i}^{\sigma}(\omega) & =G_{\sigma}(\omega) \\
& =\frac{1-n_{\bar{\sigma}}}{\omega+\mu_{\sigma}-J_{\sigma}(\omega)}+\frac{n_{\bar{\sigma}}}{\omega+\mu_{\sigma}-U-J_{\sigma}(\omega)}
\end{aligned}
$$

The Larkin irreducible part $\Xi_{\sigma}^{-1}=\omega+\mu_{\sigma}-\Sigma_{\sigma}$ is determined by the following expression

$$
\begin{aligned}
\Xi_{\sigma}^{-1}(\omega) & =\left[\frac{1-n_{\bar{\sigma}}}{\omega+\mu_{\sigma}-J_{\sigma}(\omega)}+\frac{n_{\bar{\sigma}}}{\omega+\mu_{\sigma}-U-J_{\sigma}(\omega)}\right]^{-1} \\
& +J_{\sigma}(\omega) .
\end{aligned}
$$

This approximation is obtained in our approach when the projecting constant $\varphi_{\sigma}$ and the function $R_{\sigma}(\omega)$ in (45) are neglected.

To compare analytically results of the alloy-analogy approximation and the Hubbard-I approximation we can examine the equations in the infinite- $U$ limit. The selfconsistency condition gives $J_{\sigma}=t_{\sigma}^{2} G_{\sigma}$ on the Bethe lattice. Thus, in this case, we have the following expression for the particle spectrum:

$$
\rho_{\sigma}^{\mathrm{AA}}(\omega)=\frac{1}{2 \pi t_{\sigma}^{2}} \sqrt{4 t_{\sigma}^{2}\left(1-n_{\bar{\sigma}}\right)-\left(\omega+\mu_{\sigma}\right)^{2}}
$$

when $\left|\omega+\mu_{\sigma}\right|<2 t_{\sigma} \sqrt{1-n_{\bar{\sigma}}}$ and zero otherwise. The height and width of this spectral band depend on the concentration $n_{\bar{\sigma}}$ and they are scaled proportionally to $\sqrt{1-n_{\bar{\sigma}}}$ when $n_{\bar{\sigma}}$ changes. Therefore, the bandwidth of the subbands (for finite $U$ ) is wider in the alloy-analogy approximation at half-filling Fig. 1(A,C) as well as off half-filling Fig. 1(B,D). In Fig. 2 the effect of wider subbands is illustrated by the dependence of the chemical potential $\mu_{A}$ on the concentration $n_{A}$ calculated using the particle density of states (47).

\section{ANALYTIC PROPERTIES AT MOTT TRANSITION}

We consider here the metal-insulator transition at halffilling. It is known that for the Hubbard model at zero 


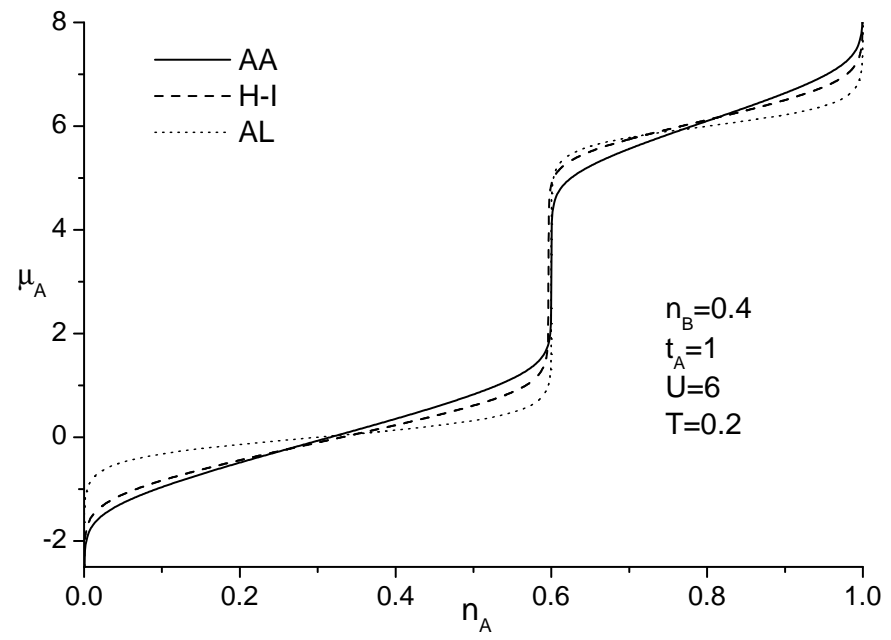

FIG. 2: The chemical potential as a function of the concentration in the alloy-analogy approximation (solid line) on the Bethe lattice compared to the Hubbard-I approximation (dashed line) and the atomic limit $t_{\sigma} \rightarrow 0$ (dotted line).

temperature the transition occurs when $\operatorname{Im} \Sigma_{\sigma}(0)=0$ and the real part has the following low-frequency form [1]

$$
\operatorname{Re} \Sigma_{\sigma}\left(\omega+\mathrm{i} 0^{+}\right)=U / 2+(1-1 / Z) \omega+\mathrm{O}\left(\omega^{3}\right)
$$

corresponding to Fermi liquid behaviour, where $Z$ defines the quasiparticles weight. Such self-energy gives particle densities of states with a quasiparticle peak at the Fermilevel that has the same height for different $U$ up to the critical value $U_{\mathrm{c}}$ [26]. The gap opens only when $Z \rightarrow 0$ and in the metallic phase the relation $G_{\sigma}(0) J_{\sigma}(0)=-1$ is satisfied.

However, for finite temperatures the Fermi-liquid state breaks near the transition point $U_{\mathrm{c}}$. Therefore, $\Sigma_{\sigma}(0)$ has a nonzero imaginary part [30], and the limit $Z \rightarrow 0$ cannot indicate the transition. In this case, the density of states at Fermi-level continuously tends to zero. At high enough temperatures, the Mott transition turns into a crossover from a bad metal to a bad insulator [46].

Another limit of the asymmetric Hubbard model (the Falicov-Kimball model) at half-filling does not have a central quasiparticle peak in the spectrum 20, 22]. The alloy-analogy, Hubbard-III and GH3 approximations also cannot describe the Fermi liquid behaviour at half-filling. Hence, these approximations can be applied to the investigation of high temperature properties of the system when the quasiparticle features disappear.

Thus, to investigate the continuous transition in the asymmetric Hubbard model, we derive some properties following from the particle hole-symmetry.

\section{A. Exact relations between $G_{\sigma}(0), J_{\sigma}(0)$ and $\Xi_{\sigma}(0)$ at continuous crossover at half-filling}

In the case of the symmetric noninteracting density of states

$$
\rho_{\sigma}^{0}(\varepsilon)=\rho_{\sigma}^{0}(-\varepsilon)
$$

leading to the particle-hole symmetry with the properties

$$
G_{\sigma}(\omega)=-G_{\sigma}(-\omega), \quad J_{\sigma}(\omega)=-J_{\sigma}(-\omega)
$$

of the single-particle Green's function and the coherent potential at half-filling, the following relations can be proven

$$
\begin{gathered}
\lim _{U \rightarrow U_{\mathrm{c}}^{-}} \frac{J_{\sigma}(0)}{G_{\sigma}(0)}=\int_{-\infty}^{+\infty} \varepsilon^{2} \rho_{\sigma}^{0}(\varepsilon) \mathrm{d} \varepsilon, \\
\lim _{U \rightarrow U_{\mathrm{c}}^{-}} G_{\sigma}(0) \Xi_{\sigma}^{-1}(0)=1
\end{gathered}
$$

which take place at zero frequency when the continuous metal-insulator transition (crossover) is approached from below (when the gap opens in the particle spectrum at $\omega=0)$.

Proof. Let us consider general properties of the Green's functions $\left(G_{\sigma}, J_{\sigma}\right)$ and the total irreducible part $\Xi_{\sigma}$ following from (62) at zero frequency. These functions can be written in the Lehmann representation

$$
F(\omega)=-\frac{1}{\pi} \lim _{\eta \rightarrow 0^{+}} \int_{-\infty}^{+\infty} \frac{\operatorname{Im} F\left(\omega^{\prime}+\mathrm{i} \eta\right) \mathrm{d} \omega^{\prime}}{\omega-\omega^{\prime}} .
$$

This representation shows that due to the symmetry (62) the functions at $\omega=0$ are pure imaginary, and there are three possible cases: (i) $F(0)=0$; (ii) $\operatorname{Re} F(0)=0$, $\operatorname{Im} F(0)=$ const; (iii) the pole of $F(\omega)$ in the real axis at $\omega=0$.

An imaginary part of $G_{\sigma}\left(\omega \pm 0^{+}\right)$defines the particle spectrum (47). When the gap continuously opens the single-particle Green's function tends to zero at $\omega=0$. It means that $\Xi_{\sigma}^{-1}(0)$ is pure imaginary

$$
\lim _{\eta \rightarrow 0^{+}} \Xi_{\sigma}^{-1}( \pm \mathrm{i} \eta)= \pm \mathrm{i} B_{\sigma}, \quad B_{\sigma}>0
$$

and the gap opens (in the non-Fermi liquid state) only when $B_{\sigma}$ continuously increases up to infinity, i.e., the self-energy diverges [1, 30, 47].

The coherent potential can be expressed from (16) as a function of $G_{\sigma}$ and the total irreducible part $\Xi_{\sigma}$ :

$$
\frac{J_{\sigma}}{G_{\sigma}}=\frac{\Xi_{\sigma}^{-1} G_{\sigma}-1}{G_{\sigma}^{2}}
$$

Using that the noninteracting density of states is symmetric (61) and normalized

$$
\int_{-\infty}^{+\infty} \rho_{\sigma}^{0}(\varepsilon) \mathrm{d} \varepsilon=1
$$


we have:

$$
G_{\sigma}=\mp \mathrm{i} B_{\sigma} \int_{-\infty}^{+\infty} \frac{\rho_{\sigma}^{0}(\varepsilon) \mathrm{d} \varepsilon}{\varepsilon^{2}+B_{\sigma}^{2}}
$$

and

$$
G_{\sigma} J_{\sigma}=\Xi_{\sigma}^{-1} G_{\sigma}-1=-\int_{-\infty}^{+\infty} \frac{\varepsilon^{2} \rho_{\sigma}^{0}(\varepsilon) \mathrm{d} \varepsilon}{\varepsilon^{2}+B_{\sigma}^{2}}
$$

from (17).

When the critical value $U_{\mathrm{c}}$ is approached from below, the limit $B_{\sigma} \rightarrow+\infty$ should be considered. In this limit, inserting (69) and (70) into (67) leads to the result (63).

To prove (64), we consider three different cases depending on behaviour of the function $\rho_{\sigma}^{0}(\varepsilon)$ at large energies.

1. If an average kinetic energy per particle is finite, the limit

$$
\lim _{\varepsilon \rightarrow \infty} \varepsilon^{2} \rho_{\sigma}^{0}(\varepsilon)=0
$$

is satisfied giving the dependence $G_{\sigma} J_{\sigma} \sim B_{\sigma}^{-2}$ (70) at large $B_{\sigma}$. It means that $J_{\sigma}(0) \rightarrow 0$ at the transition.

2. When the density of states has the power-law tails of order $\varepsilon^{-2}$

$$
\lim _{\varepsilon \rightarrow \infty} \varepsilon^{2} \rho_{\sigma}^{0}(\varepsilon)=\text { const }>0,
$$

the limiting behaviour $G_{\sigma} J_{\sigma} \sim B_{\sigma}^{-1}$ is realized at the transition. In this case a finite imaginary part of $J_{\sigma}(0)$ remains when $B_{\sigma} \rightarrow \infty$, and the ratio $J_{\sigma}(0) / G_{\sigma}(0)$ tends to infinity [63).

An example of such a function is the Lorentzian density of states describing a lattice with longrange hopping along coordinate axes

$$
\rho_{\sigma}^{0}(\varepsilon)=\frac{t_{\sigma}}{\pi\left(\varepsilon^{2}+t_{\sigma}^{2}\right)} .
$$

This density of states always gives a constant value for the coherent potential:

$$
\lim _{\eta \rightarrow 0^{+}} J_{\sigma}(\omega \pm \mathrm{i} \eta)=\mp \mathrm{i} t_{\sigma} .
$$

3. The last case is when $\rho_{\sigma}^{0}(\varepsilon) \sim \varepsilon^{-n}, n \in(1,2)$ at large energy $\varepsilon$. The integrals (68) and (70) are convergent only when $n>1$. Such power-law tails give the following limiting behaviour $G_{\sigma} J_{\sigma} \sim$ $1 /\left(B_{\sigma}\right)^{n-1}$ and $J_{\sigma} \sim\left(B_{\sigma}\right)^{2-n}$ at large $B_{\sigma}$.

All these cases show that the relation

$$
\lim _{U \rightarrow U_{\mathrm{c}}^{-}} G_{\sigma}(0) J_{\sigma}(0)=-0
$$

is satisfied proving the relation (64).

\section{B. Critical value $U_{\mathrm{c}}$ for the Mott transition within GH3 approximation with symmetric density of states}

Due to (63), we have

$$
J_{\sigma}(0)=W_{\sigma}^{2} G_{\sigma}(0) / 4
$$

at the Mott transition, where $W_{\sigma}$ is the effective halfbandwidth of the unperturbed density of states $\rho_{\sigma}^{0}$ :

$$
W_{\sigma}=2\left(\int_{-\infty}^{+\infty} \varepsilon^{2} \rho_{\sigma}^{0}(\varepsilon) \mathrm{d} \varepsilon\right)^{1 / 2} .
$$

Due to $G_{\sigma}(0) \Xi_{\sigma}^{-1}(0)=1(64)$ at the transition, inserting (76) into (52) yields a set of equations:

$$
\begin{aligned}
& \lim _{U \rightarrow U_{\mathrm{c}}^{-}} \frac{U^{2} G_{A}(0)}{W_{A}^{2} G_{A}(0)+2 W_{B}^{2} G_{B}(0)}=1, \\
& \lim _{U \rightarrow U_{\mathrm{c}}^{-}} \frac{U^{2} G_{B}(0)}{W_{B}^{2} G_{B}(0)+2 W_{A}^{2} G_{A}(0)}=1 .
\end{aligned}
$$

The ratio

$$
\eta=\lim _{U \rightarrow U_{\mathrm{c}}^{-}} \frac{G_{A}(0)}{G_{B}(0)}
$$

is excluded from the set of equations, and we have the following equation for $U_{\mathrm{c}}$

$$
U_{\mathrm{c}}^{4}-\left(W_{A}^{2}+W_{B}^{2}\right) U_{\mathrm{c}}^{2}-3 W_{A}^{2} W_{B}^{2}=0
$$

with the solution

$$
U_{\mathrm{c}}=\sqrt{\frac{W_{A}^{2}+W_{B}^{2}+\left(W_{A}^{4}+W_{B}^{4}+14 W_{A}^{2} W_{B}^{2}\right)^{1 / 2}}{2}} .
$$

If we put $W_{B}=0$, the expression (82) describes the Mott-type transition in the alloy-analogy approximation (which is exact for the Falicov-Kimball model) giving well known results: $U_{\mathrm{c}}=W_{A}=2 t_{A}$ for the Bethe lattice and $U_{\mathrm{c}}=\sqrt{2} t_{A}$ for the hypercubic lattice. In the case of the standard Hubbard model $\left(W_{A}=W_{B}=W\right)$, we have the result $U_{\mathrm{c}}=\sqrt{3} W$ corresponding to the Hubbard-III approximation at half-filling (see Refs. 11, 16 for reviews of the limits: the standard Hubbard model and the FalicovKimball model).

\section{RESULTS AND DISCUSSION}

There are two different pairs of parameters $\left(n_{A}, \mu_{A}\right.$ and $\left.n_{B}, \mu_{B}\right)$ in the asymmetric Hubbard model which have to be determined from the thermodynamic equilibrium. Thus, various thermodynamic regimes may be considered when different pairs of the thermodynamic parameters are fixed: $\left(\mu_{A}, \mu_{B}\right),\left(\mu_{A}, n_{B}\right),\left(n_{A}, n_{B}\right)$, etc. It is known that there are possible phase separations at low 
temperatures in the case of the Falicov-Kimball model [34, 48, 49] and in the general case of the asymmetric Hubbard model 6]. Besides the segregated phases, the long-range antiferromagnetic-type ordering is possible at low temperatures [3].

Since the investigation of the asymmetric Hubbard model is a very complicated problem, we restrict our analysis to temperatures higher than the critical temperatures of thermodynamic instabilities. Thus, the problem is reduced to the investigation of a band structure and metal-insulator transitions at constant particle concentrations.

The simplest approximations such as the Hubbard-I and alloy-analogy approximations describe the band of particles generated by the particle hopping and the interaction with particles of another sort. These approximations can describe an effect of the transfer parameter $t_{A}$ on the spectrum $\rho_{B}(\omega)$ only via the concentration $n_{A}$, i.e., when the concentrations are fixed the band $\rho_{B}(\omega)$ does not depend on $t_{A}$, and for $t_{A} \neq 0, t_{B}=0$ the approximate spectrum $\rho_{B}(\omega)$ has a form of two delta-peaks. However, the alloy-analogy approximation is exact only for itinerant particles in the Falicov-Kimball model when another sort of particles is localized. Thus, this approximation can give simple reasonable results for dependence $\mu_{\sigma}=\mu_{\sigma}\left(n_{\sigma}, n_{\bar{\sigma}}\right)$ in the limit of small values of $n_{\bar{\sigma}}$ or $t_{\bar{\sigma}}$.

The generalization of the Hubbard-III approximation (GH3) gives the total irreducible part and the single-site Green's function as a functional of the coherent potentials of both sorts of particles (see equations (44)-(46)). Unlike the alloy-analogy approximation, GH3 described broadening of the band by the interaction with particles of another sort, and it gives a finite bandwidth for localized particles in the Falicov-Kimball model. In general, the spectral density in the GH3 approximation is temperature dependent; such an approximation can be applied for systems with different values of the transfer parameters (both $t_{A}=t_{B}$ and $t_{A} \neq t_{B}$ ) and various particle concentrations. However, the approximation has some restrictions. In Ref. 43, GH3 was applied to the calculation of the chemical potential and spectrum of localized particles in the Falicov-Kimball model. It was shown that the approximation gives better results when the concentration of itinerant particles is low.

In the symmetric case of half-filling the approximate density of states is independent of temperature (Fig. [3), because the projecting coefficients and the coefficients of the integral terms in $R_{\sigma}(\omega)$ (46) are equal to zero. In the Falicov-Kimball model at half-filling, the approximation (53) can be compared with the exact results [20, 22]. We find that the approximation gives a better correspondence with the exact curves at high temperatures, and the best correspondence is for high $U$ when the exact results weakly depend on temperature 43]. Fig. 3(A) shows that the density of states of heavy particles $B$ (the case of $t_{B}<t_{A}$ ) is in the form of a single peak for the low interaction $U$. For the higher interaction strength Fig. 3(B,C), the spectrum $\rho_{B}(\omega)$ has two subbands with

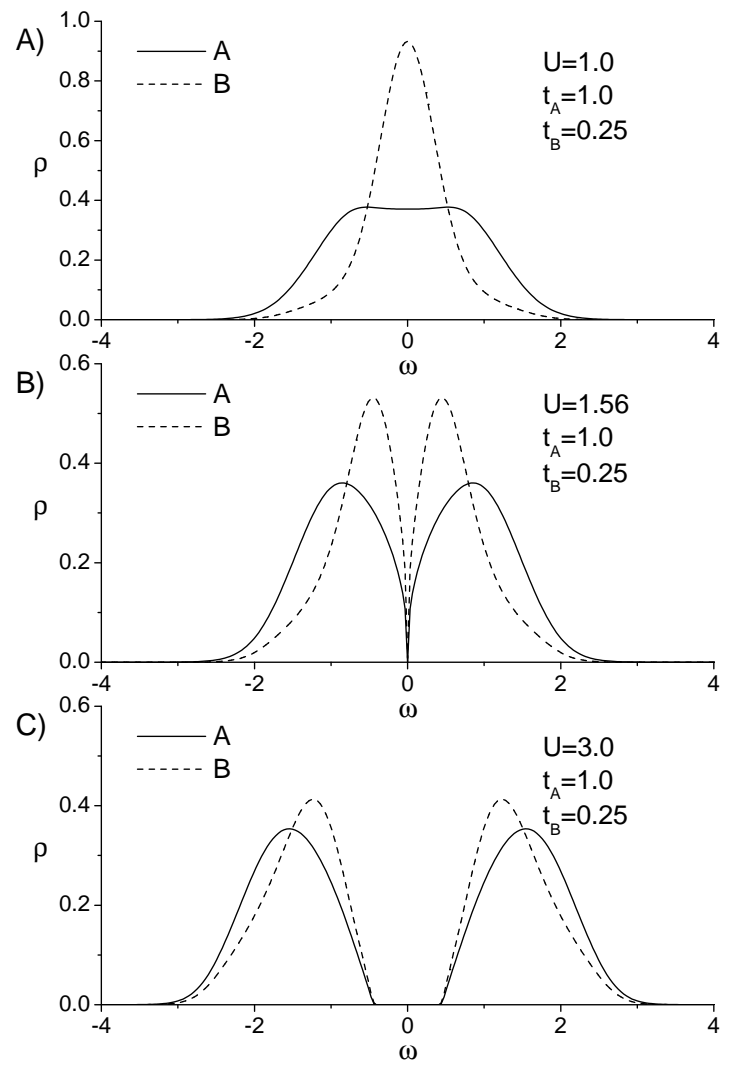

FIG. 3: Densities of states of particles in the asymmetric Hubbard model within the GH3 approximation for various values of $U$ at half-filling on the hypercubic lattice.

peaks which are closer to the centre $(\omega=0)$ than in the spectrum of light particles $\rho_{A}(\omega)$. This agrees with the results obtained for the Falicov-Kimball model when $t_{B}=0$.

The particle-hole symmetry at half-filling simplifies the investigation of the problem. Such symmetry requires the divergence $(1 / \omega)$ of the self-energy at zero frequency when the gap opens continuously [30, 47]. Thus, the limit behaviour (63), (64) of the Green's function and the coherent potential at the transition is proven analytically, which allows us to perform some analytic analysis.

In Fig. 3] the transition with the opening gap is illustrated for the asymmetric Hubbard model within the GH3 approximation. There are only continuous transitions. This agrees with the fact that the Mott transition has to be of a continuous type at high temperatures for the standard Hubbard model [25, 30], and in the case of the Falicov-Kimball model the gap opens continuously with increasing $U[16]$. The spectra of both types of particles have the same bandwidth in the approximation. This is because it cannot describe tails of the subbands that raise with the temperature increase as it is for localized particles in the Falicov-Kimball model [20, 22]. Since the effect of temperature broadening of the band is not captured, it can be predicted that the approximation 


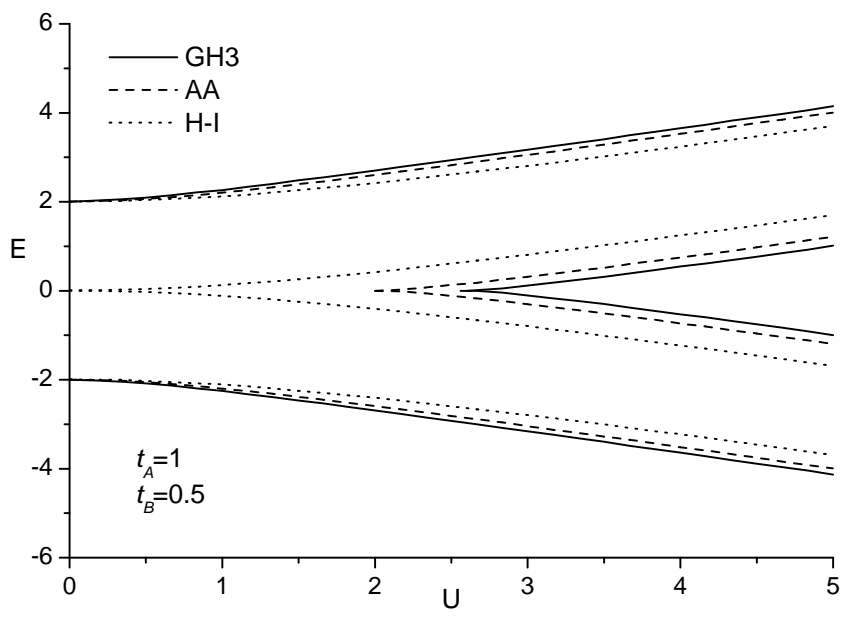

FIG. 4: Dependence of the energy band edges on the repulsion strength $U$ at half-filling $n_{A}=n_{B}=1 / 2$ on the Bethe lattice: $t_{A}=1, t_{B}=0.5$. The solid line is the GH3 approximation, the dashed and dotted lines are the alloy-analogy and Hubbard-I approximations, respectively.

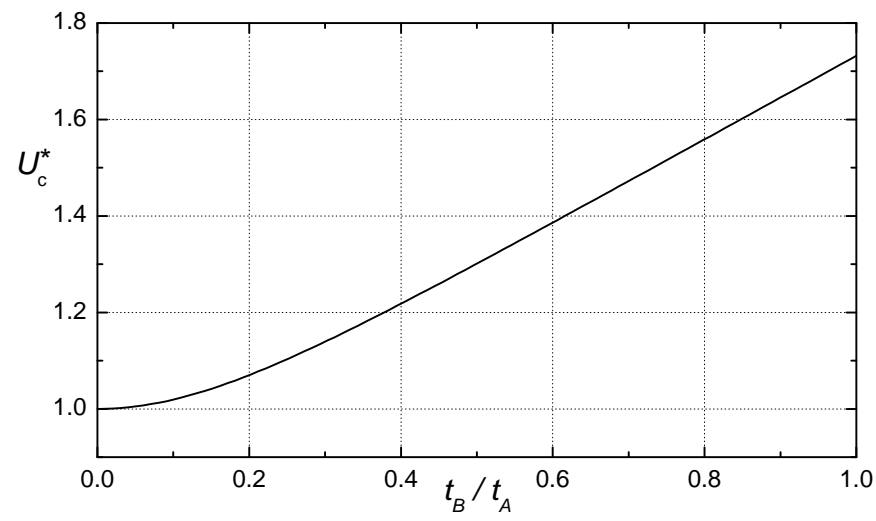

FIG. 5: Scaled critical value $U_{\mathrm{c}}^{*}=U_{\mathrm{c}} / W_{A}$ (83) as a function of $t_{B} / t_{A}$ within the GH3 approximation at half-filling.

underestimate the critical value $U_{\mathrm{c}}$.

The energy band edges as a function of $U$ within various approximations on the Bethe lattice are displayed in Fig. [4 In the Hubbard-I approximation the spectrum is always split having the gap and two subbands. The alloyanalogy approximation is exact for the band of itinerant particles when $t_{B}=0$ and it gives the critical interaction constant $U_{\mathrm{c}}^{\mathrm{AA}}=2 t_{A}$. The GH3 approximation describes the band which is broadened by simultaneous contribu- tion of the hopping of both types of particles, and the critical value $U_{\mathrm{c}}>U_{\mathrm{c}}^{\mathrm{AA}}$. Because of the particle-hole symmetry at half-filling the critical value $U_{\mathrm{c}}$ can be calculated analytically in the GH3 approximation and it is given by the expression (82), see Fig. [5 The obtained result can be applied to various lattices (hypercubic, Bethe, etc.) when the energy is scaled in the following way:

$$
U_{\mathrm{c}}^{*}=\frac{U_{\mathrm{c}}}{W_{A}}=\frac{U_{\mathrm{c}}}{2}\left(\int_{-\infty}^{+\infty} \varepsilon^{2} \rho_{A}^{0}(\varepsilon) \mathrm{d} \varepsilon\right)^{-1 / 2} .
$$

It is common practice to normalize $U$ by a typical kinetic energy $\left(W_{A}\right)$ investigating the Mott transition in the standard Hubbard model 11]. In this case, such normalization shows that the scaled result does not depend on a form of the noninteracting density of states. For the Lorentzian density of states (73) with long-range hopping an average kinetic energy per particle tends to infinity $\left(W_{A} \rightarrow \infty\right)$, and as it was noted in Ref. 50 , this requires an infinite interaction $U$ to drive the system insulating.

\section{CONCLUSIONS}

The generalization of the Hubbard-III approximation is obtained for the asymmetric Hubbard model using the equation of motion approach. This method combines in a unified framework the description of the band structure of the Falicov-Kimball model and the standard Hubbard model. In general, the approach can be used to calculate the particle spectrum in the system with the different particle concentrations at various temperatures, and it gives nontrivial results for the spectrum of localized particles in the Falicov-Kimball model.

The self-consistency condition connecting mutually the single-site Green's function and the coherent potential gives the simple result (63) when the metal-insulator transition occurs at half-filling on the lattice with a symmetric noninteracting density of states. As a result, we have the expression (82) in the GH3 approximation for the critical value of $U$ that provides a universal solution for various lattices and different values of the transfer parameters.

The approximation has some restrictions. Thus, to describe the system at half-filling with the proper temperature dependence or the quasi particle peak, the approach needs further improvement in calculating the mass operator by including higher order corrections.
[1] L. M. Falicov and J. C. Kimball, Phys. Rev. Lett. 22, 997 (1969).

[2] J. Hubbard, Proc. R. Soc. London, Ser. A 276, 238 (1963).

[3] A. N. Kocharian and G. R. Reich, J. Appl. Phys. 76, 6127 (1994).
[4] A. N. Kocharian and G. R. Reich, Physica B 206-207, 719 (1995).

[5] C. Gruber, R. Kotecký, and D. Ueltschi, J. Phys. A: Math. Gen. 33, 7857 (2000).

[6] D. Ueltschi, J. Stat. Phys. 116, 681 (2004).

[7] G. Fáth, Z. Domański, and R. Lemański, Phys. Rev. B 
52, 13910 (1995).

[8] T. Fukui and N. Kawakami, Phys. Rev. B 58, 16051 (1998).

[9] C. A. Macedo and A. M. C. de Souza, Phys. Rev. B 65, 153109 (2002).

[10] W. Metzner and D. Vollhardt, Phys. Rev. Lett. 62, 324 (1989).

[11] A. Georges, G. Kotliar, W. Krauth, and M. J. Rozenberg, Rev. Mod. Phys. 68, 13 (1996).

[12] E. Müller-Hartmann, Z. Phys. B 74, 507 (1989).

[13] V. I. Anisimov, A. I. Poteryaev, M. A. Korotin, A. O. Anokhin, and G. Kotliar, J. Phys.: Condens. Matter 9, 7359 (1997).

[14] K. Held, I. A. Nekrasov, G. Keller, V. Eyert, N. Blümer, A. K. McMahan, R. T. Scalettar, T. Pruschke, V. I. Anisimov, and D. Vollhardt, Psi-k Newsletter 56, 65 (2003).

[15] A. Georges, cond-mat/0403123 (2004).

[16] J. K. Freericks and V. Zlatić, Rev. Mod. Phys. 75, 1333 (2003).

[17] U. Brandt and C. Mielsch, Z. Phys. B 75, 365 (1989).

[18] U. Brandt and C. Mielsch, Z. Phys. B 79, 295 (1990).

[19] U. Brandt and C. Mielsch, Z. Phys. B 82, 37 (1991).

[20] U. Brandt and M. P. Urbanek, Z. Phys. B 89, 297 (1992).

[21] V. Zlatić, J. K. Freericks, R. Lemański, and G. Czycholl, Phil. Mag. B 81, 1443 (2001).

[22] J. K. Freericks, V. M. Turkowski, and Zlatić, Phys. Rev. B 71, 115111 (2005).

[23] R. M. Noack and F. Gebhard, Phys. Rev. Lett. 82, 1915 (1999).

[24] W. Krauth, Phys. Rev. B 62, 6860 (2000).

[25] J. Schlipf, M. Jarrell, P. G. J. van Dongen, N. Blümer, S. Kehrein, T. Pruschke, and D. Vollhardt, Phys. Rev. Lett. 82, 4890 (1999).

[26] R. Bulla, Phys. Rev. Lett. 83, 136 (1999).

[27] M. J. Rozenberg, R. Chitra, and G. Kotliar, Phys. Rev. Lett. 83, 3498 (1999).

[28] R. Bulla and M. Potthoff, Eur. Phys. J. B 13, 257 (2000).

[29] Y. Ōno, R. Bulla, A. C. Hewson, and M. Potthoff, Eur.
Phys. J. B 22, 283 (2001).

[30] R. Bulla, T. A. Costi, and D. Vollhardt, Phys. Rev. B 64, 045103 (2001).

[31] M. P. Eastwood, F. Gebhard, E. Kalinowski, S. Nishimoto, and R. M. Noack, Eur. Phys. J. B 35, 155 (2003).

[32] D. Vollhardt, K. Held, G. Keller, R. Bulla, T. Pruschke, I. A. Nekrasov, and V. I. Anisimov, J. Phys. Soc. Japan 74, 136 (2005).

[33] G. Kotliar, J. Phys. Soc. Japan 74, 147 (2005).

[34] I. V. Stasyuk and O. B. Hera, Condens. Matter Phys. 6, 127 (2003).

[35] I. V. Stasyuk, Condens. Matter Phys. 3, 437 (2000).

[36] I. V. Stasyuk and A. M. Shvaika, Ukr. J. Phys. 47, 975 (2002).

[37] T. Herrmann and W. Nolting, Phys. Rev. B 53, 10579 (1996).

[38] J. Hubbard, Proc. R. Soc. London, Ser. A 281, 401 (1964).

[39] H. O. Jeschke and G. Kotliar, Phys. Rev. B 71, 085103 (2005).

[40] A. M. Shvaika, Phys. Rev. B 67, 075101 (2003).

[41] W. Metzner, Phys. Rev. B 43, 8549 (1991).

[42] D. Vollhardt, N. Blümer, K. Held, M. Kollar, J. Schlipf, and M. Ulmke, Z. Phys. B 103, 283 (1997).

[43] I. V. Stasyuk and O. B. Hera, unpublished, submitted to Phys. Rev. B, preprint cond-mat/0502669 (2005).

[44] J. K. Freericks and L. M. Falicov, Phys. Rev. B 41, 2163 (1990).

[45] B. Velický, S. Kirkpatrick, and H. Ehrenreich, Phys. Rev. 175, 747 (1968).

[46] M. J. Rozenberg, G. Kotliar, and X. Y. Zhang, Phys. Rev. B 49, 10181 (1994).

[47] C. Gros, Phys. Rev. B 50, 7295 (1994).

[48] J. K. Freericks, C. Gruber, and N. Macris, Phys. Rev. B 60, 1617 (1999).

[49] B. M. Letfulov, Eur. Phys. J. B 11, 423 (1999).

[50] A. Georges, G. Kotliar, and Q. Si, Int. J. Mod. Phys. B 6, 705 (1992). 